\title{
In-plane effects on segmented-mirror control
}

\author{
Douglas G. MacMynowski, ${ }^{1, *}$ Lewis C. Roberts, Jr., ${ }^{2}$ J. Chris Shelton, ${ }^{2}$ \\ Gary Chanan, ${ }^{3}$ and Henri Bonnet ${ }^{4}$ \\ ${ }^{1}$ Control and Dynamical Systems, California Institute of Technology \\ 1200 East California Boulevard, Pasadena, California 91125, USA \\ ${ }^{2}$ Jet Propulsion Laboratory, California Institute of Technology, Pasadena, California 91109, USA \\ ${ }^{3}$ Physics and Astronomy, University of California, Irvine, Irvine, California 92697, USA \\ ${ }^{4}$ European Southern Observatory, Garching, Germany \\ *Corresponding author: macmardg@cds.caltech.edu
}

Received 14 December 2011; revised 26 January 2012; accepted 27 January 2012; posted 27 January 2012 (Doc. ID 159097); published 11 April 2012

\begin{abstract}
Extremely large optical telescopes are being designed with primary mirrors composed of hundreds of segments. The "out-of-plane" piston, tip, and tilt degrees of freedom of each segment are actively controlled using feedback from relative height measurements between neighboring segments. The "in-plane" segment translations and clocking (rotation) are not actively controlled; however, in-plane motions affect the active control problem in several important ways, and thus need to be considered. We extend earlier analyses by constructing the "full" interaction matrix that relates the height, gap, and shear motion at sensor locations to all six degrees of freedom of segment motion, and use this to consider three effects. First, in-plane segment clocking results in height discontinuities between neighboring segments that can lead to a global control system response. Second, knowledge of the in-plane motion is required both to compensate for this effect and to compensate for sensor installation errors, and thus, we next consider the estimation of in-plane motion and the associated noise propagation characteristics. In-plane motion can be accurately estimated using measurements of the gap between segments, but with one unobservable mode in which every segment clocks by an equal amount. Finally, we examine whether in-plane measurements (gap and/or shear) can be used to estimate out-of-plane segment motion; these measurements can improve the noise multiplier for the "focus-mode" of the segmented-mirror array, which involves pure dihedral angle changes between segments and is not observable with only height measurements. (C) 2012 Optical Society of America
\end{abstract}

OCIS codes: $110.6770,110.1080$.

\section{Introduction}

Optical telescopes larger than about $8 \mathrm{~m}$ are enabled by building a segmented primary mirror, an approach pioneered by the Keck telescopes. While the Keck telescopes each have 36 segments, the design for the Thirty Meter Telescope (TMT) shown in Fig. 1 has 492 [1], the $39 \mathrm{~m}$ European Extremely Large Telescope (E-ELT) design [2] has 798, and concepts have been proposed with many more segments [3].

$1559-128 \mathrm{X} / 12 / 121929-10 \$ 15.00 / 0$

(C) 2012 Optical Society of America
The "out-of-plane" piston, tip, and tilt degrees of freedom of each segment are typically controlled using feedback from edge sensors as at Keck [4], while the remaining three "in-plane" translation and clocking motions are left uncontrolled and constrained by the passive support system. (By "in-plane" we mean the motion of each segment in the plane tangent to the local optical prescription.) Nonhexagonal segmentation is also possible [5]; here we focus on the hexagonal case used in optical telescope designs. (We do not consider segmented-mirrors such as for the James Webb Space Telescope, where real-time feedback with edge sensors is not required.) 


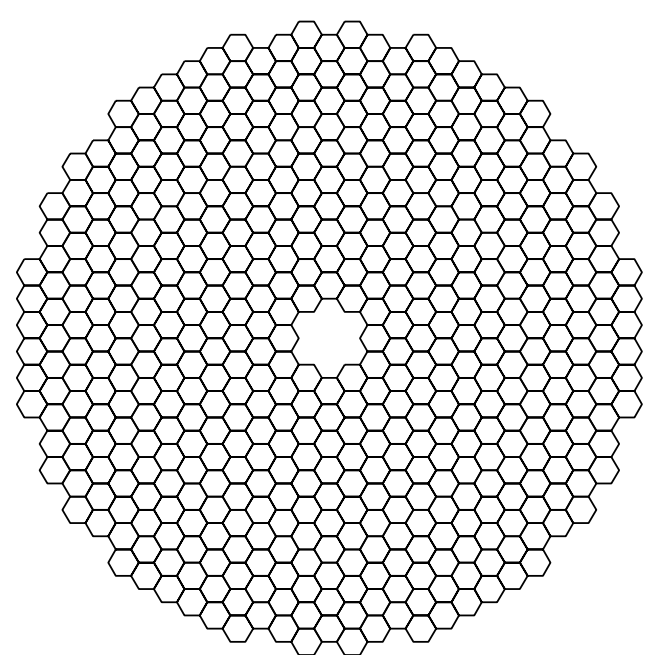

Fig. 1. Hexagonal segmentation geometry used in examples. Each segment has three actuators, and two sensors on each intersegment edge; for the 492-segment geometry shown (that of TMT) this leads to a total of 1476 actuators and 2772 sensors.

Control of the out-of-plane degrees of freedom relies on an interaction matrix (the "A-matrix") between the controlled motion (or equivalently actuator commands) and the sensor response. This matrix is constructed from geometry [6], and a singular value decomposition has proven useful in understanding the estimation of segment motion and the propagation of sensor noise. Understanding the effects of the in-plane motions on the control of the out-of-plane degrees of freedom relies on constructing the interaction matrix between all six degrees of freedom of segment motion and the resulting relative translations at the sensor locations, including not only the relative height discontinuity used by the control system but also the gap between the

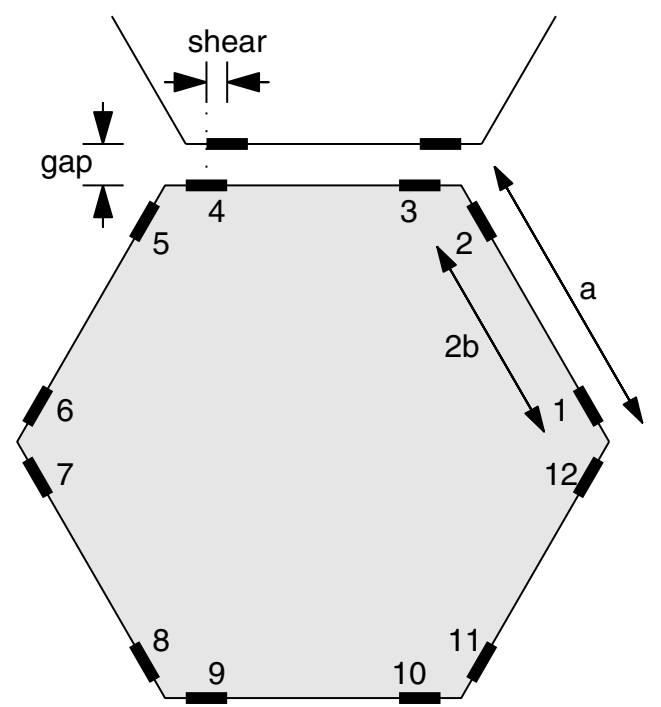

Fig. 2. Sensor geometry of an individual segment, and definition of gap and shear at sensor location. Sensors on a segment are numbered clockwise, and the segment radius $a$ and distance $b$ from edge center to sensor are indicated. segments and the shear motion parallel to the edge [see Fig. 2 for illustration of gap and shear motion]. The next section describes the construction of this matrix; this extends the results in [6]. In-plane motion is relevant to the control of the remaining degrees of freedom for three reasons.

First, for a curved (rather than flat) mirror, rotation about the segment-center normal (commonly called clocking) or in-plane translations of an individual segment result in nonzero height discontinuities between the segment and its neighbors. A control system that seeks to minimize the rootmean-square (rms) sensor height discontinuity will propagate errors across the primary mirror [7]. Nonzero sensor installation errors also result in cross-talk between the gap and shear motion at the sensor location and the sensor height reading; this can be a significant effect if not corrected. These effects can be minimized by estimating the in-plane motion, and using this to correct the desired sensor readings based on a calibration algorithm [8].

This leads to the second coupling between inplane motion and out-of-plane control: the ability to estimate the in-plane motion using measurements of only the gap at sensor locations (as planned for TMT), or with both gap and shear measurements (as planned for the E-ELT). The measurement accuracy is quantified in terms of the noise multiplier that relates the rms estimation error to the sensor noise standard deviation.

Finally, given that some in-plane information is required to estimate the in-plane motion, we consider whether this information can also be used to improve the estimates of out-of-plane motion. This is only relevant for focus-mode, which involves a change in the radius of curvature of the mirror, and also some small wavefront scalloping due to the resulting mismatch between the mirror and segment radius of curvature. This response pattern involves pure dihedral angle changes between neighboring segments and is thus unobservable from height-sensors alone. However, in addition to being measurable from dihedral (as at Keck or planned for TMT), focus-mode also yields a gap signature; the analysis shows that disentangling this from the gap signature caused by in-plane motion requires either dihedral sensitivity or shear measurements in addition to gap [7].

We first describe the construction of the full interaction matrix in Section 2 , and then consider each of the three ways described above that in-plane motion affects control of the segmented-mirror. Extensions to standard noise multiplier calculations that are needed for analysis are described in the appendix.

\section{Full Interaction Matrix}

\section{A. Description}

The A-matrix described in control analyses of segmented primary mirrors [6] relates the vector of height and dihedral response $y_{h}$ at segment edge 
sensor locations to the vector $z$ of mirror segment displacements at the actuator locations, so $y_{h}=A_{h z} z$. If the actuators are perfect position actuators, then the matrix can equivalently be described as the sensor response to quasi-static actuator commands. The control algorithm uses this information, together with the difference between actual and desired sensor readings, to determine an estimate $\hat{z}$ of the segment motion (with unobservable global piston, tip, tilt projected out), and then determines the control required to drive the estimated displacement towards zero. [Note that because the actuators push perpendicularly to the local surface prescription, then the three (nearly) singular modes of $A_{h z}$ are not quite global piston, tip, and tilt; nonetheless, these patterns are not estimated or controlled.] A singular value decomposition of $A_{h z}$ has been useful in understanding the properties of this matrix [6]. The output signal from each sensor is a linear combination of a signal generated by a height change and a signal due to the change in dihedral angle (relative rotation about the shared segment edge) [8]. The relative sensitivity between these has units of length (i.e., $\mathrm{m} /$ radian), and is denoted $L_{\text {eff }}$; (at Keck, $L_{\text {eff }}=$ $55 \mathrm{~mm}$ corresponds to a physical moment arm). Without dihedral sensitivity, focus-mode is unobservable (corresponding to a singular value of zero). For realistic choices of dihedral sensitivity, focus-mode remains the least-observable deflection pattern (smallest singular value) after global piston, tip, and tilt. The next least-observable modes (relatively large ratio of rms surface motion to resulting $\mathrm{rms}$ edge discontinuity [9]) are spatially smooth and similar to Zernike basis functions.

The full interaction matrix relates all three displacements at the sensor location (height combined with dihedral, gap, and shear) to all six possible rigid-body deflections of each mirror segment. The segmentnormal motion $z$ can be described as before by the motion at each of the three actuator locations, while the remaining "in-plane" motion $w$ is described by radial and azimuthal translations of the segment along the surface that describes the optical prescription, and the clocking about the segment-center normal. It is convenient to scale the clocking by a length (here we use the segment radius $a$ ) so that $z$ and $w$ both have units of length, and the interaction matrix is dimensionless. Thus with $y_{h}, y_{g}$, and $y_{s}$ as the height (combined with dihedral), gap, and shear respectively at the sensor locations, with corresponding noise $\eta_{h}, \eta_{g}, \eta_{s}$, then

$$
\left[\begin{array}{l}
y_{h} \\
y_{g} \\
y_{s}
\end{array}\right]=\left[\begin{array}{ll}
A_{h z} & A_{h w} \\
A_{g z} & A_{g w} \\
A_{s z} & A_{s w}
\end{array}\right]\left[\begin{array}{c}
z \\
w
\end{array}\right]+\left[\begin{array}{l}
\eta_{h} \\
\eta_{g} \\
\eta_{s}
\end{array}\right] .
$$

For TMT there are $N=492$ segments, $n_{a}=3 N=$ 1476 actuators, and $n_{s}=2772(<6 N)$ edge-sensor locations, so the full interaction matrix has dimension $(2772 \times 3)$ by $(492 \times 6)$. The subblock $A_{h z} \in$ $\mathbb{R}^{n_{s} \times n_{a}}$ is the standard $A$-matrix described earlier.
The full interaction matrix has rank $6 N-6$ with unobservable degrees of freedom corresponding to the six rigid-body motions of the primary mirror.

\section{B. Calculation}

For an idealized flat mirror, there is no coupling between in-plane and out-of-plane motion, so $A_{h w}=$ $A_{g z}=A_{s z}=0$. The nonzero values of these subblocks result from the mirror curvature and asphericity, and thus depend on the particular telescope design. However, the blocks $A_{h z}, A_{g w}$, and $A_{s w}$ are nonzero even for a flat mirror, and while there will be some small deviations when the specific details of the geometry are taken into account, the dominant effects can be understood from generic formulas. For a hexagonally segmented mirror, the formulas for computing $A_{h z}$ are given in [6], and thus we first extend this to the blocks $A_{g w}$ and $A_{s w}$. After that, we describe the more general algorithm for computing the full interaction matrix for any specific telescope geometry.

To construct $A_{g w}$ and $A_{s w}$, we first construct the $12 \times 3$ matrix $g$ that relates in-plane motion of a single segment to the gap displacements at the sensor locations on that segment, and similarly the single segment $12 \times 3$ matrix $s$ that relates in-plane motion of the segment to shear displacements at the sensor locations on that segment. Thus with sensors on an individual segment numbered clockwise starting from the $x$-axis [see Fig. 2], and clocking scaled by $a$,

$$
g=\left[\begin{array}{ccc}
-\sqrt{3} / 2 & -1 / 2 & -b / a \\
-\sqrt{3} / 2 & -1 / 2 & b / a \\
0 & -1 & -b / a \\
0 & -1 & b / a \\
\sqrt{3} / 2 & -1 / 2 & -b / a \\
\sqrt{3} / 2 & -1 / 2 & b / a \\
\sqrt{3} / 2 & 1 / 2 & -b / a \\
\sqrt{3} / 2 & 1 / 2 & b / a \\
0 & 1 & -b / a \\
0 & 1 & b / a \\
-\sqrt{3} / 2 & 1 / 2 & -b / a \\
-\sqrt{3} / 2 & 1 / 2 & b / a
\end{array}\right] \text { and }
$$

These are illustrated in Fig. $\underline{3}$. The gap between segments has an unambiguous sign convention (increased gap is positive), while there is a choice 

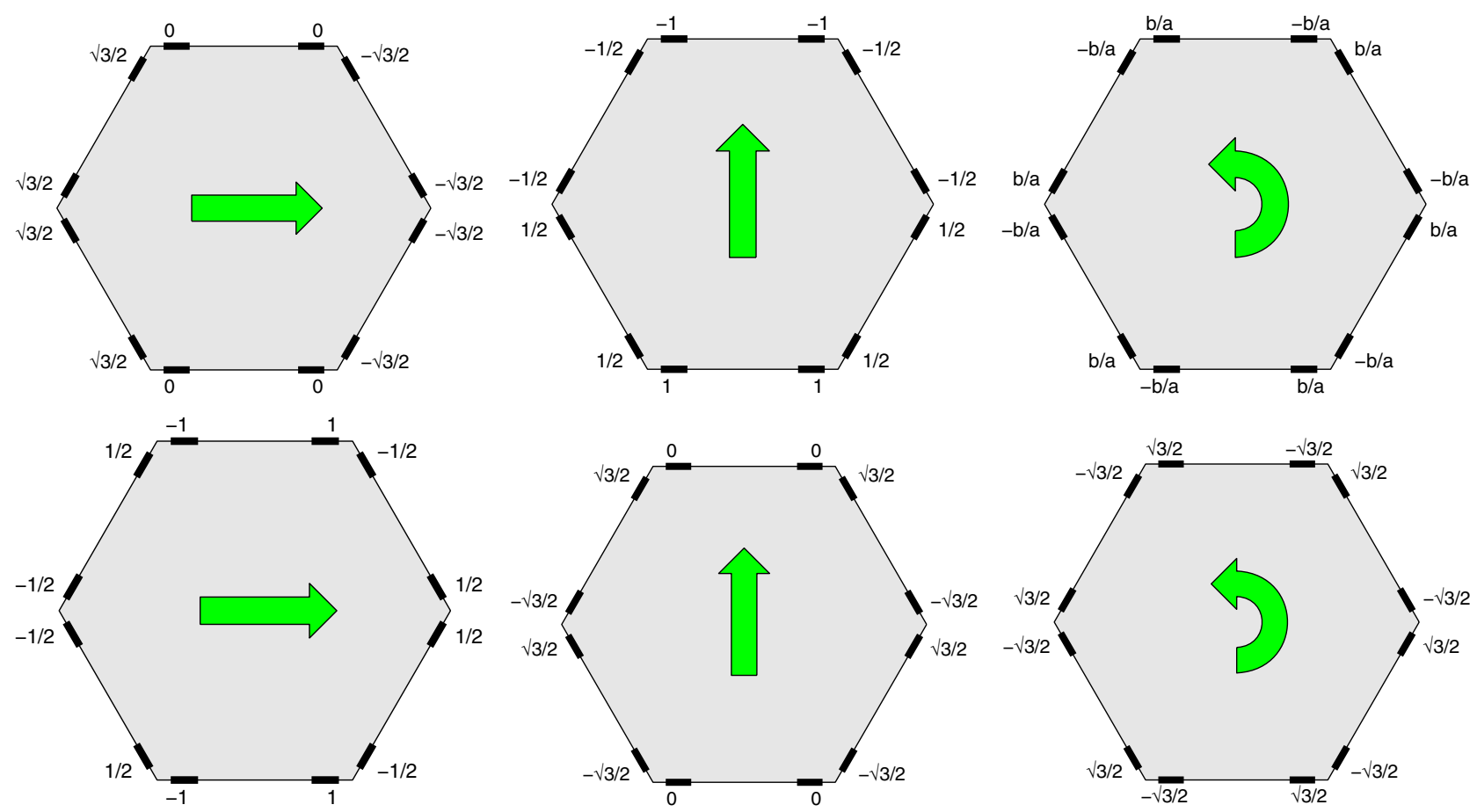

Fig. 3. (Color online) Constructing the single-segment $12 \times 3$ matrices $g$ and $s$ that relate in-plane $x$ and $y$ translation and clocking to the gap (top row) and shear motion (bottom row) at the sensor locations; these correspond to Eq. (6).

to make in defining the sign for shear. Using a coordinate system fixed to each sensor with direction $\xi$ pointing along the segment edge in a clockwise direction [see Fig. 2], we define the sign convention to be positive for positive $\xi$ for even-numbered sensors and negative for odd-numbered sensors. Note that each sensor location corresponds to an even number on one segment and an odd number on the neighbor; this sign convention allows all segments to be equivalent. Once the local $(12 \times 3) g$ and $s$ matrices have been defined, it is then straightforward bookkeeping to expand these into the global matrices $A_{g w}$ and $A_{s w}$; these are both $n_{s} \times n_{a}$ matrices.

Next, consider the matrices that couple in-plane and out-of-plane motion, $A_{h w}, A_{g z}$, and $A_{s z}$. These matrices depend on the details of the segmented-mirror geometry, and thus we describe the algorithm for constructing them, rather than providing explicit formulas as above for $A_{g w}$ and $A_{s w}$. First, since a mirror that is not flat cannot be tiled by regular hexagons, the starting point for construction of the interaction matrix is the design choice of segment shape and position, and the actuator and sensor locations on each segment. The interaction matrix is obtained by assuming that the segments, with sensors attached, move as rigid bodies. The lateral constraint mechanism of each segment ensures that "out-ofplane" motion due to actuation acts about a pivot point that is along the segment-center normal. (For a curved geometry, the segments are not perfect hexagons and thus the "segment-center" is only welldefined from the design choice of the pivot point.) "In-plane" translations and clocking are then defined relative to this pivot point, completing the definition of the coordinate system for the six degrees of freedom of segment motion. Once coordinate systems are also defined for each sensor, then the interaction matrix follows from three-dimensional transformations from segment motion to sensor motion. The sensor coordinate system is chosen attached to one segment (the "drive" side) with the height, gap, and shear defined relative to the local segment surface at the sensor location; the motion of the other segment (the "sense" side) must also be described in this same coordinate system.

The reason that $A_{h w}, A_{g z}$, and $A_{s z}$ are nonzero in general is as follows. In-plane motion along a curved mirror causes a height response at the sensor location (nonzero $A_{h w}$ ) due to the change in the radial location of the sensor. The change in gap at the sensor location that is caused by out-of-plane motion (the matrix $A_{g z}$ ) results from the difference in the direction between the segment-center normal and the normal vector at the sensor location. For example, if both segments move a distance $h$ along their respective segment-normals, then the gap between segments decreases by $g=(\sqrt{3} / 2)\left(a / R_{0}\right) h$, where $R_{0}$ is the radius of curvature. The shear motion at sensor locations that is caused by out-of-plane motion $\left(A_{s z}\right)$ similarly results from a nonzero projection of the motion along the segment-center normal and the shear direction at the sensor location (along the mirror surface, tangent to the segment edge), for a sensor that is not in the center of an edge.

\section{Preliminary Analysis}

The effects of in-plane motion are evaluated in the following using an influence matrix constructed for 
the 492-segment geometry in Fig. 1, and smaller segment-arrays obtained by removing segments beyond some specified radius. We chose $b / a=0.36$, $a=0.72$, and a sensor dihedral sensitivity of $L_{\text {eff }} \simeq 24 \mathrm{~mm}$. The value of $L_{\text {eff }}$ only matters in Section 5 . In addition to computing the influence matrix for a spherical-mirror, we have also computed the influence matrix for the $f / 1$ Ritchey-Chretien geometry of TMT in order to validate that none of the conclusions herein depend significantly on the choice of spherical or aspheric geometry.

The signatures of a few relevant response patterns are shown in Fig. 4. "Focus-mode" we define as the singular vector of $\bar{A}_{h z}$ that has the least nonzero observability (i.e., other than global piston, tip, and tilt); this could instead be defined in terms of Zernike focus at the actuator locations, and would have no significant impact on conclusions. As noted earlier, if $L_{\text {eff }}=0$ (no dihedral sensitivity), then this mode is unobservable with only height sensors. The second mode shape shown in Fig. $\underline{4}$ is "breathing-mode," where all of the segments expand radially along the surface describing the optical prescription. This pattern produces mostly a gap signature but also some shear and height changes; the last of these would be zero on a flat mirror. Finally, "torsion-mode" we define from the singular value decomposition of the matrix $A_{g w}$ that relates in-plane motion to the gaps at sensor locations. This matrix has rank $n_{a}-4$, with torsion mode unobservable in addition to the three in-plane rigid-body motions. For a spherical mirror, this motion involves equal clocking of every segment, illustrated in Fig. $\underline{5}$. This produces pure shear at the sensor locations, and zero gap motion (to first order). With a nonspherical mirror, there is a very slight change in the mode shape; this does not have a significant impact on any conclusions.

\section{Response to In-Plane Motion}

For a curved, rather than flat, mirror, there is a height discontinuity between neighboring segments that results from segment in-plane motion (that is, $A_{h w} \neq 0$ ). For translations, the response of the control system to the height discontinuity is primarily to introduce a tip/tilt of the translated segment, which partially compensates the optical effect. However, for segment clocking, the control algorithm can increase the surface rms error by trying to correct the apparent motion; the out-of-plane displacement that results is the estimated out-of-plane motion $\hat{z}=$ $A_{h z}^{\#} y_{h}$ based on assuming that the measured height discontinuities $y_{h}=A_{h w} w$ are due to out-of-plane motion:

$$
\hat{z}=A_{h z}^{\#} A_{h w} w
$$

where we use the notation $(\cdot)^{\#}$ to denote the pseudoinverse. This is plotted in Fig. $\underline{6}$ for a representative single-segment clocking case and for torsion mode. Torsion mode results in the control system erroneously introducing nonzero focus-mode due to the edge height discontinuity.

Furthermore, in addition to the response in Eq. (3) for ideal sensors, small sensor misalignments result in cross-axis sensitivity to in-plane motion, again leading to a global out-of-plane control system response to in-plane motions. For realistic installation tolerances, this latter effect is much more significant.

\section{Estimation of In-Plane Motion}

To correct for the effects above, the in-plane motion must be estimated. Calibrating the sensor installation errors does not require $w$ directly, but requires knowledge of both the gap and shear at sensor locations. In principle, the in-plane motion could be estimated using only gap measurements $y_{g}$, only shear measurements $y_{s}$, or using both; if only one is measured, the other must be estimated.

Other than the effect of the unobservable torsion mode, the vector of in-plane motion $w$ can be estimated from the gap measurements, and this used to estimate the shear at sensor locations [8]. This allows calibration of the sensor response to both gap and shear motion using only gap measurements, with errors due to the unobservable torsion mode, and due to the propagation of gap-measurement noise. Furthermore, the gap measurements can be improved by projecting out the nonphysical component of the measurement noise:

$$
\begin{gathered}
\hat{w}=A_{g w}^{\#} y_{g}, \\
\hat{y}_{s}=A_{s w} \hat{w}=A_{s w} A_{g w}^{\#} y_{g},
\end{gathered}
$$
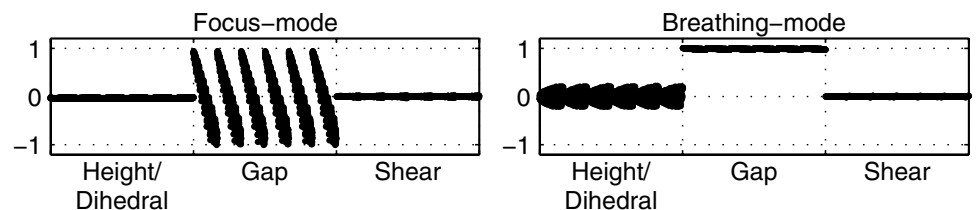

Fig. 4. Each subplot shows, for a particular deflection pattern of the mirror, the resulting response at each of the $n_{s}$ sensor locations: the height (mixed with dihedral; first set of $n_{s}=2772$ points), gap (second set of 2772 points), and shear motion (third set). In each case, the response is normalized by the maximum response. The pattern of motion within a given sensor type depends on the sensor numbering convention; it is the relative response between height, gap, and shear for each deflection pattern that is of interest. The three deflection patterns shown are focus-mode (left), breathing-mode (center), corresponding to pure translation of every segment radially along the optical prescription, and torsion-mode (right), corresponding to uniform clocking of every segment. Focus-mode also produces a nearly-uniform dihedral signature that is small compared to the gap signature. 


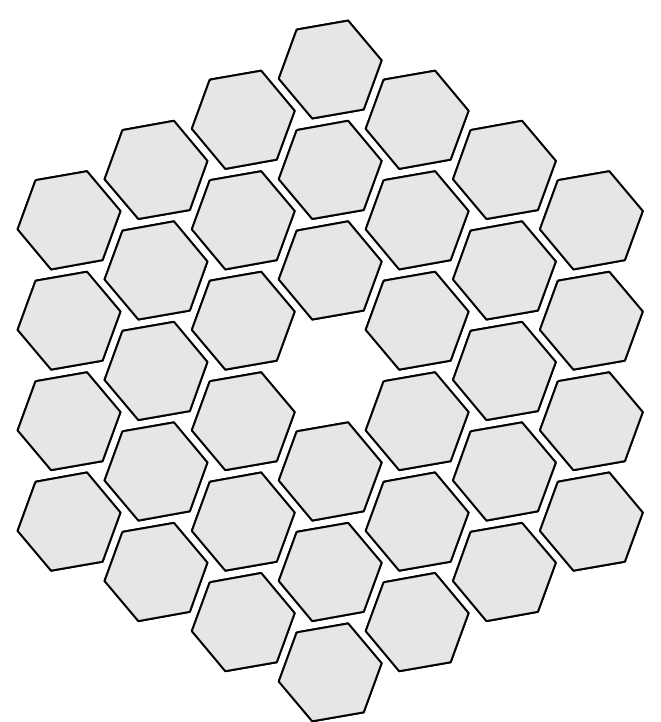

Fig. 5. Torsion mode pattern (illustrated for Keck segmentation geometry); equal clocking of every segment produces only shear at sensor locations, and no change in the gaps between segments.

$$
\hat{y}_{g}=A_{g w} \hat{w}=A_{g w} A_{g w}^{\#} y_{g} .
$$

Similar equations can be used to estimate in-plane motion from shear measurements, or from both gap and shear. Of course if both gap and shear are measured, it is not essential to introduce the added complication of improving the estimates analogously to Eq. (6). Implicit in the above equations is that the out-of-plane motion is controlled, and therefore small compared to the in-plane motion. If this is not true, then some of the gap or shear motion may be due to out-of-plane motion, and the full vector of six degree of freedom segment motion can be estimated from all of the available sensor information.

The quality of the estimate is described by a noise multiplier that relates the rms estimation error to the sensor noise standard deviation. Some additional observations regarding the calculation of noise multipliers beyond what is presented in [6] are given in the appendix. Noise multipliers for the in-plane estimation problem are tabulated for the 492-segment
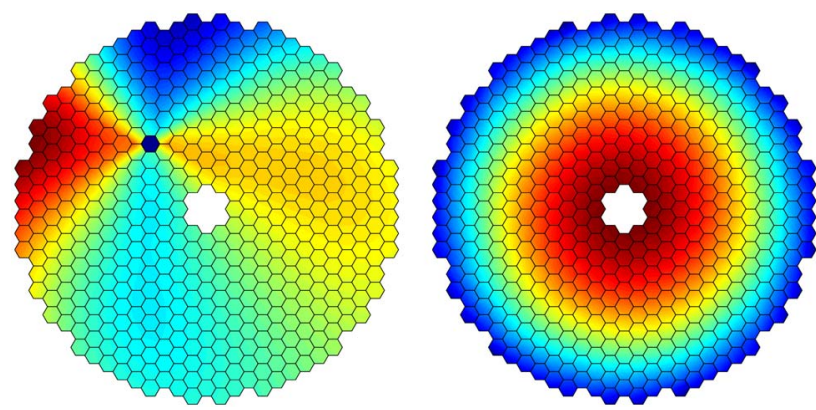

Fig. 6. (Color online) Response pattern that results from the control system canceling the sensor height readings that result from in-plane clocking of a single segment (left; the rms surface error is $\sim 40 \mathrm{~nm} / \mathrm{mrad}$ ) and due to torsion mode (right; the rms surface error is $6.8 \mu \mathrm{m} / \mathrm{mrad})$. example in Table 1; unlike the noise multiplier from height-sensor noise to actuator response, these noise multipliers are not a strong function of the number of segments in the mirror, as shown in Fig. 7 . The noise multipliers for individual modes (see appendix for definition) are shown in Fig. 8. The values shown for the gap-measurement case in both the table and figures neglect the unobservable torsion mode. With shear information alone, while there are no unobservable modes, there are many poorly sensed modes. (An example is shown in Fig. $\underline{9}$; large-scale patterns of in-plane motion do not result in any appreciable shear.) This leads to a poor estimate of inplane motion, and thus of gap. Note that the noise multiplier from gap or shear noise to gap or shear estimates is dimensionless $(\mathrm{nm} / \mathrm{nm})$; however the definition of a noise multiplier for in-plane motion $\hat{w}$ depends on the choice of relative units between translations and rotations. As noted, here we measure these both in meters by scaling the rotation by segment radius $a$ so that the overall noise multiplier is dimensionless. One can also compute the noise multiplier separately for translations and clocking (included in Table 1); note that the estimation errors on these are correlated. Similarly, using gap to estimate shear results in correlated estimation errors between $\hat{y}_{s}$ and $\hat{y}_{g}$.

Finally, note that when using gap measurements to estimate in-plane motion, torsion mode is not observable from the gaps alone but is weakly observable from combined gap and height measurements. Alternatively, it is also observable with even a single shear sensor, and a few (6-7) randomly distributed shear measurements is sufficient to make the error multiplier for this mode comparable to that of the next least-observable mode.

\section{Estimation of Focus-Mode}

Given that gap or shear measurements are required to estimate the in-plane motion, the final question considered here is whether these in-plane

Table 1. Noise Multipliers for the 492-Segment Example for Estimating In-Plane Motion from Measurements of Gap Only, Shear Only, or Gap and Shear Combined ${ }^{a}$

\begin{tabular}{llllll}
\hline & $\hat{w}$ & $\widehat{x y}$ & $\hat{\theta}$ & $\hat{y}_{g}$ & $\hat{y}_{s}$ \\
\hline Gap only & 1.02 & 0.78 & 1.9 & 0.73 & 2.23 \\
Shear only & 372 & 450 & 117 & 128 & 0.73 \\
Gap \& shear & 0.53 & 0.58 & 0.55 & 0.49 & 0.54 \\
\hline
\end{tabular}

${ }^{a}$ In addition to the vector of physical motion $w$ and the unmeasured sensor variables, the noise multiplier is also computed for the improvement in knowledge of measured variables that is obtained by projecting the overdetermined sensor set onto the physically possible response. The noise multiplier is dimensionless (or equivalently $\mathrm{nm} / \mathrm{nm}$ ) for displacements such as the in-plane translations (labeled here " $x y$ ") or estimates of gap and shear motion $\hat{y}_{g}$ and $\hat{y}_{s}$. The noise multiplier for segment clocking (labeled here " $\theta$ ") is given in $\mathrm{rad} / \mathrm{m}$, while the noise multiplier for the full vector of physical motion depends on the choice of measuring translations or sensor outputs in meters, and $\theta$ in radians scaled by the segment radius $a$. 


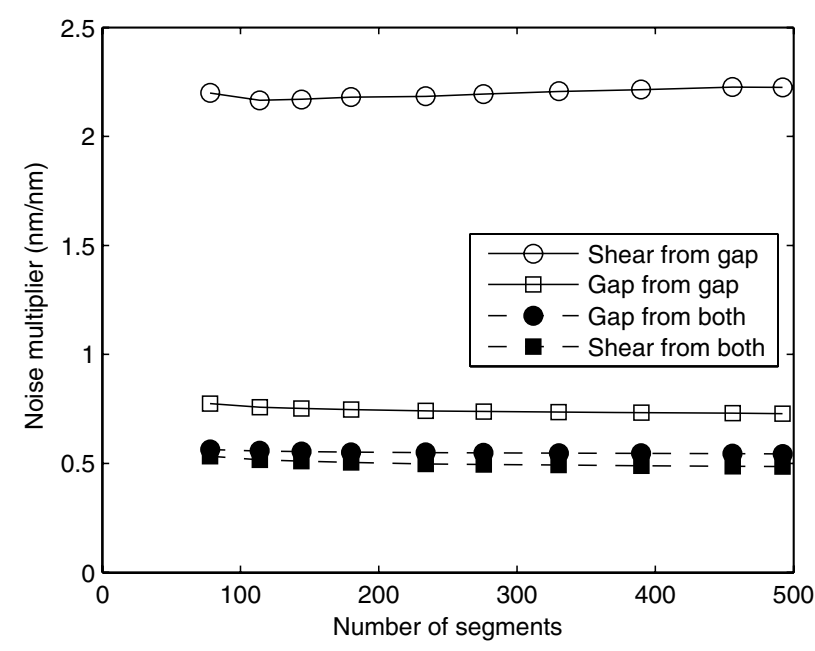

Fig. 7. In-plane noise multiplier scaling as a function of number of segments, for estimating gap and shear motion at sensor locations from either gap information alone, or from both gap and shear. The estimation errors using only shear information are significantly larger, as shown in Table $\underline{1}$.

measurements can also be useful in estimating outof-plane motion, and focus-mode in particular (the remaining in-plane degrees of freedom are observable with height measurements). While focus-mode can be estimated from the measurements $y_{h}$ as long as the dihedral sensitivity $L_{\text {eff }}$ is nonzero, the noise multiplier can be quite large for small $L_{\text {eff }}$; furthermore, focus-mode corresponds to a nearly-uniform dihedral change, and its estimation is therefore particularly sensitive to any correlated drift across all sensors.

Focus-mode noise multipliers can be evaluated for various sensor combinations; see the appendix regarding how these are calculated. For a single sensor type, the noise multiplier gives the dimensionless ratio of rms estimation error to $\mathrm{rms}$ sensor noise. With multiple sensor types, the answer also depends

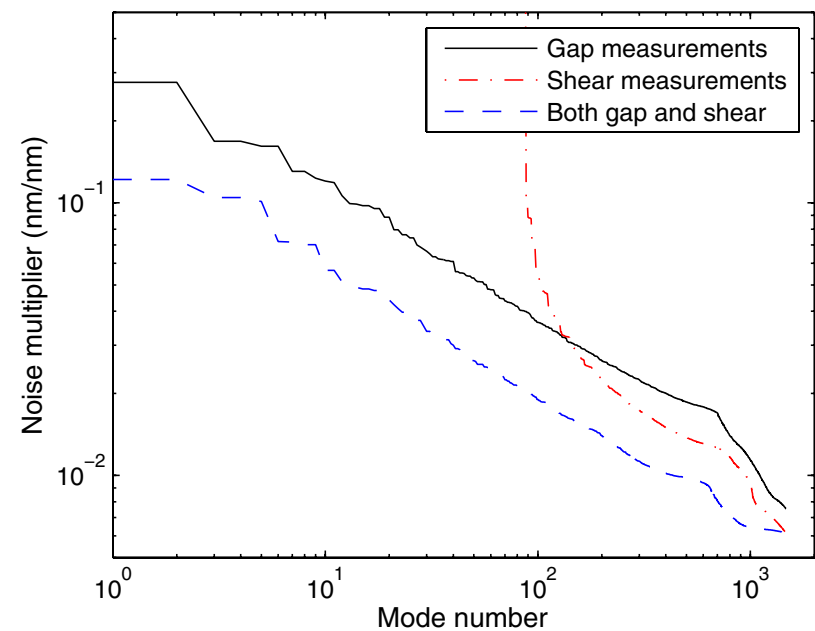

Fig. 8. (Color online) Modal noise multiplier for estimating inplane motion from measurements of gap alone, shear alone, or both gap and shear. The unobservable torsion mode using only gap sensors is not shown (the noise multiplier is infinite for this mode). Units are $\mathrm{nm} / \mathrm{nm}$ with clocking scaled by length $a$.

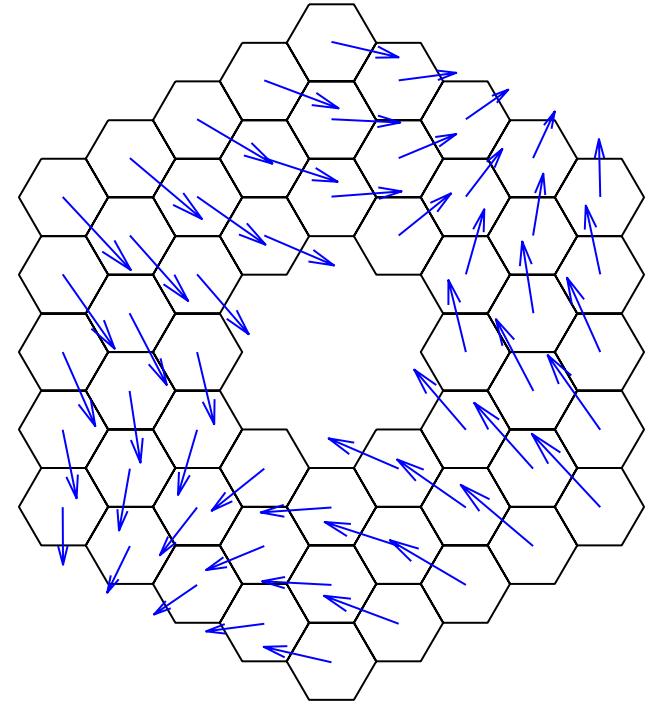

Fig. 9. (Color online) Least-observable pattern of in-plane motion with shear sensors (for a 54-segment array); there is also some very small segment clocking not shown.

on the ratio of rms sensor noise on one type of sensor to another. Figure 10 plots the noise multiplier for several sensor combinations as a function of the ratio of gap and shear measurement noise to the height/ dihedral measurement noise. (Of course, many other combinations are possible, but this is sufficient to illustrate the behavior.)

Despite the large gap signature, focus-mode is not observable using only gap sensors (or equivalently, only gap plus idealized height sensors that have zero dihedral sensitivity). In order to estimate focus-mode in the case of zero dihedral sensitivity, both gap and shear information is required in order to distinguish the gap signature of focus-mode from that of in-plane motion. However, the only practical case in which shear information is useful in estimating focus-mode

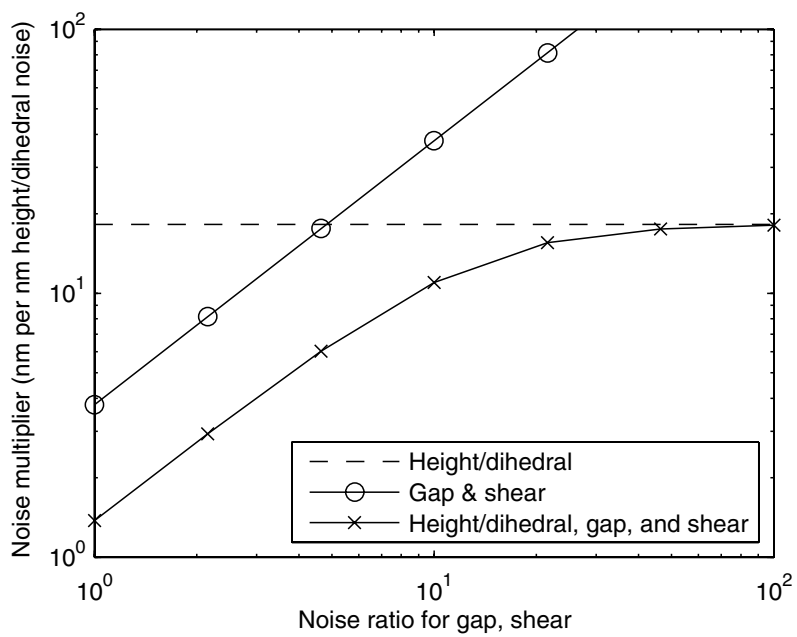

Fig. 10. Noise multiplier for estimating focus-mode, per $\mathrm{nm}$ of height/dihedral noise, for 492-segment example with $L_{\text {eff }}=$ $24 \mathrm{~mm}$. Both the gap and shear sensor noise standard deviations are assumed to be higher than the height/dihedral noise by the factor on the abscissa. 
is if the dihedral sensitivity is zero. The curves for combining height/dihedral with shear information are not plotted in Fig. 10, because at the noise levels considered here, and with the dihedral sensitivity considered here, shear information is not useful as a complement to dihedral. The same is true if both height/dihedral and gap are available; this curve follows the same trajectory as the full sensor information case plotted. Shear measurements alone are insufficient to estimate focus-mode, and thus the three curves in Fig. 10 capture all of the relevant combinations of sensor types.

For $L_{\text {eff }}=24 \mathrm{~mm}$ used here, the noise multiplier using dihedral sensitivity alone is roughly $18(\mathrm{~nm} / \mathrm{nm})$; this scales inversely with $L_{\text {eff }}$ and increases with the number of segments [6]. Gap and shear measurements alone give a noise multiplier of $\sim 4$; this scales inversely with the number of segments. For the combined cases, the noise multiplier is close to that of gap and shear alone for small gap/ shear noise, and the same as that for height/dihedral measurements alone if the gap/shear noise is large. The plot is similar if the shear noise is assumed to be the same as the height/dihedral noise, and only the gap noise increased; the ability to estimate focusmode from in-plane measurements is dominated by the noise on the gap sensing.

Note in the above that if focus-mode has nonzero projection onto the unobservable subspace of the interaction matrix, then the noise multiplier would be infinite. In practice, there is always some nonzero projection due to numerical calculations. Furthermore, there are typically several almostunobservable modes, and these may have some very small projection onto focus-mode. In this case, there is potential for some subjectivity in defining the focus-mode noise multiplier; we include sufficient basis vectors to project onto $99.5 \%$ of the mean-square value of focus-mode. However, this means that the noise multiplier is only accurate to about two significant digits.

There are also differences in the use of gap and shear information in terms of noise propagation from correlated sensor noise to the focus-mode estimate. Because focus-mode corresponds to nearly uniform dihedral across all sensors, correlated sensor noise results in a significant estimation error, almost equal to the worst-case analysis in Eq. (A10) in the appendix. The gap-signature of focus-mode is nearly zero-mean, and hence correlated noise on gap sensors produces almost no estimation error in focus-mode.

\section{Conclusions}

While only the out-of-plane degrees of freedom of a segmented mirror are typically controlled, the remaining in-plane degrees of freedom affect the control in several ways. Analysis requires construction of the full interaction matrix between all six degrees of freedom of segment motion and the height (mixed with dihedral), gap and shear relative motions at sensor locations. This extends the earlier construction of the subblock of this interaction matrix between actuator motion and sensor height, which is essential to control [6].

In-plane motion leads to height discontinuities between neighboring segments that, if not accounted for, result in a response from the control system that can increase the overall rms surface error; this effect is straightforward to compute from the full interaction matrix. Furthermore, sensor installation errors lead to cross-axis sensitivity to in-plane motions (gap and shear); because in-plane motions are allowed to be much larger than the out-of-plane motion, either this effect needs to be corrected or very tight installation tolerances maintained.

In order to correct for both of these effects, the inplane motion needs to be estimated, and both the gap and shear at sensor locations known. However, this does not require that both gap and shear be measured. With only gap measurements, there is one unobservable mode that we label "torsion mode," involving equal clocking of every segment, which produces pure shear motion. Except for the contribution from this unobservable mode, the in-plane motion, and thus the shear at sensor locations, can be estimated. The noise multiplier is of order $2 \mathrm{~nm}$ estimation error per $\mathrm{nm}$ sensor noise, and does not scale with the number of segments. The noise multiplier for estimating in-plane motion from shear sensors alone is much higher than that from gap measurements.

Given that at least gap measurements are required to estimate the in-plane motion, it is also useful to understand whether this additional information can complement height and dihedral information in estimating the out-of-plane segment motion. Gap measurements are useful in addition to dihedral for estimating focus-mode if they are sufficiently accurate (of order 5-10 times less accurate than the height/dihedral measurement for the dihedral sensitivity assumed here), but focus-mode is not observable with gap measurements alone, and either dihedral sensitivity or shear is required.

The TMT Project gratefully acknowledges the support of the TMT partner institutions. They are the Association of Canadian Universities for Research in Astronomy (ACURA), the California Institute of Technology and the University of California. This work was supported as well by the Gordon and Betty Moore Foundation, the Canada Foundation for Innovation, the Ontario Ministry of Research and Innovation, the National Research Council of Canada, the Natural Sciences and Engineering Research Council of Canada, the British Columbia Knowledge Development Fund, the Association of Universities for Research in Astronomy (AURA). and the U.S. National Science Foundation.

JCS and LCR are employed at the Jet Propulsion Laboratory, California Institute of Technology, which is operated under contract for NASA 


\section{Appendix A: Noise Multipliers}

A key aspect to the estimation problems described herein is the propagation of sensor noise to the estimate. The noise multiplier defined in [6] relates the rms estimation error to the standard deviation of sensor noise, assuming uncorrelated noise with equal variance on each sensor. We note here a few brief extensions to (i) accommodate different noise variance on different types of sensors (i.e., height, gap, shear), (ii) propagate errors in estimated positions to errors in a related quantity (such as shear at sensor locations, or the amplitude of focus-mode), and (iii) consider the propagation of correlated sensor noise in addition to uncorrelated noise.

Given measurements $y$ related to segment motion $x$ (which in general may include $w, z$, or both) by $y=A x+\eta$, with sensor noise $\eta$ that is zero-mean with covariance $R$, then the minimum-variance estimate of $x$ is given by the weighted pseudoinverse of A:

$$
\hat{x}=\lim _{\epsilon \rightarrow 0}\left(A^{T} R^{-1} A+\epsilon I\right)^{-1} A^{T} R^{-1} y,
$$

where the regularization term $\epsilon$ is required to deal with $A$ being rank-deficient. If the measurement equations are rescaled so that the sensor noise covariance is $R=\rho^{2} I$ for scalar $\rho$, then this reduces to the usual unweighted pseudoinverse. If the unscaled sensor noise is uncorrelated between all sensors $(R$ diagonal), then this corresponds to scaling the rows of both $A$ and $y$ by the standard deviation of each sensor noise term. More generally, this scaling can always be done by, for example, replacing $A$ with $R^{-1 / 2} A$ and $y$ with $R^{-1 / 2} y$. Henceforth we will assume this noise-scaled form for $A$, so that the estimate in Eq. (A1) is simply $\hat{x}=A^{\#} y$.

Using the singular value decomposition of the scaled $A$ matrix,

$$
A=U \Sigma V^{T},
$$

where $U$ and $V$ are unitary and $\Sigma$ is diagonal with entries $\sigma_{i}$, and defining the notation $\Sigma^{-1}$ to mean inverting only the nonzero (or larger than some tolerance) diagonal elements of $\Sigma$, then

$$
\hat{x}=V \Sigma^{-1} U^{T} y .
$$

It is convenient to define the error $\tilde{x}$ to be the projection of the estimation error onto the observable subspace (i.e. $\tilde{x}=\left(I-M M^{T}\right)(x-\hat{x})$, where $M$ is a basis for the nullspace of $A$ ), so that $\tilde{x}=0$ in the zero-noise limit. Then

$$
\tilde{x}=V \Sigma^{-1} U^{T} \eta
$$

and the mean-square value of $\tilde{x}$ for $\rho=1$ is

$$
\begin{aligned}
\frac{1}{n_{a}} E\left\{\tilde{x}^{T} \tilde{x}\right\} & =\frac{1}{n_{a}} \operatorname{tr}\left[E\left\{\tilde{x} \tilde{x}^{T}\right\}\right]=\frac{1}{n_{a}} \operatorname{tr}\left[V \Sigma^{-2} V^{T}\right] \\
& =\frac{1}{n_{a}} \operatorname{tr}\left[V^{T} V \Sigma^{-2}\right]=\frac{1}{n_{a}} \sum_{i} \sigma_{i}^{-2}
\end{aligned}
$$

as in [6], but now accounting for differing noise covariances by scaling the $A$ matrix rows appropriately. The noise multiplier for estimating $x$ from $y$ is the ratio of the rms estimation error to the standard deviation of sensor noise, i.e., the square root of the expression in Eq. (A5).

The noise multiplier for quantities derived from the positions, such as the estimated shear at sensor locations from the estimated in-plane motion, can be similarly derived, e.g., if $s=H x$ and defining $\tilde{s}$ similarly to $\tilde{x}$, then

$$
E\left\{\tilde{s}^{T} \tilde{s}\right\}=\operatorname{tr}\left[H V \Sigma^{-2} V^{T} H^{T}\right] .
$$

For multivariable $s$ there is no general simplification. For a scalar $f=v_{f}^{T} x$ (e.g., the amplitude of focusmode) then Eq. (A6) reduces to

$$
E\left\{(f-\hat{f})^{2}\right\}=\sum_{i}\left(\frac{v_{f}^{T} v_{i}}{\sigma_{i}}\right)^{2} .
$$

That is, the noise multiplier for focus (or any other) mode shape is simply the sum over all SVD-mode noise-multipliers, weighted by each mode's projection onto focus-mode. This will be infinite if focus-mode is not observable, i.e., if it has nonzero projection onto the unobservable subspace. If focus-mode is a singular vector from the SVD then $v_{j}=v_{f}$ for some $j$, and the formula reduces to $1 / \sigma_{j}^{2}$ (using the orthogonality of the singular vectors).

Finally, consider correlated sensor noise $\eta_{i}=\mu$ for $i \in \mathcal{Q}$ where $\mu$ is random but identical for some subset of sensors $i \in \mathcal{Q}$ of dimension $Q$. If no change were made to the estimator, then the error covariance due to the correlated noise is

$$
E\left\{(f-\hat{f})^{2}\right\}=\left(v_{f}^{T} V \Sigma^{-1} U^{T} q\right)^{2},
$$

where the vector $q$ satisfies $q_{i}=1, i \in \mathcal{Q}$ and $q_{i}=0$ otherwise. If $v_{f}=v_{j}$ then this reduces to

$$
E\left\{(f-\hat{f})^{2}\right\}=\frac{1}{\sigma_{j}^{2}}\left(u_{j}^{T} q\right)^{2} .
$$

The worst possible case is if $U_{i j}=1 / \sqrt{Q}$ for $i \in \mathcal{Q}$, giving a mean-square estimation error

$$
E\left\{(f-\hat{f})^{2}\right\}=Q / \sigma_{j}^{2} .
$$

This is approximately the case for estimating focusmode using dihedral sensitivity alone, leading to a noise multiplier a factor $\sqrt{n_{s}}$ times larger per $\mathrm{nm}$ of noise correlated across all sensors, than for uncorrelated noise with the same rms. 


\section{References}

1. J. Nelson and G. H. Sanders, "The status of the Thirty Meter Telescope project," Proc. SPIE 7012, 70121A (2008).

2. R. Gilmozzi and J. Spyromilio, "The 42m European ELT: status," Proc. SPIE 7012, 701219 (2008).

3. S. Padin, "Design considerations for a highly segmented mirror," Appl. Opt. 42, 3305-3312 (2003).

4. R. C. Jared, A. A. Arthur, S. Andreae, A. Biocca, R. W. Cohen, J. M. Fuertes, J. Franck, G. Gabor, J. Llacer, T. Mast, J. Meng, T. Merrick, R. Minor, J. Nelson, M. Orayani, P. Salz, B. Schaefer and C. Witebsky, "The W. M. Keck Telescope segmented primary mirror active control system," Proc. SPIE 1236, 996-1008 (1990).
5. T. Sebring, "The Cornell Caltech Atacama Telescope: progress and plans," Proc. SPIE 7733, 77331X (2010).

6. G. Chanan, D. G. MacMartin, J. Nelson, and T. Mast, "Control and alignment of segmented-mirror telescopes: Matrices, modes, and error propagation," Appl. Opt. 43, 1223-1232 (2004).

7. H. Bonnet, ESO Internal Report (2008)

8. C. Shelton, T. Mast, G. Chanan, J. Nelson, L. C. Roberts Jr., M. Troy, M. J. Sirota, B.-J. Seo, and D. R. MacDonald, "Advances in edge sensors for the Thirty Meter Telescope primary mirror," Proc. SPIE 7012, 701210 (2008).

9. D. G. MacMartin and G. Chanan, "Measurement accuracy in control of segmented-mirror telescopes," Appl. Opt. 43, 608-615 (2004). 